\title{
Analysis and optimization of multiple factors influencing fracturing induced stress field
}

\author{
Bing Guan ${ }^{1}(1) \cdot$ Shibin $\mathrm{Li}^{1} \cdot$ Jiran Liü ${ }^{2}$ Ligang Zhang ${ }^{1} \cdot$ Shuangqing Chen ${ }^{1}$
}

Received: 30 July 2018 / Accepted: 16 July 2019 / Published online: 25 July 2019

(c) The Author(s) 2019

\begin{abstract}
The induced stress field caused by hydraulic fracturing plays a key role in controlling the formation characteristics of fractures and is the essential prerequisite for optimal design of fracturing parameters. In order to determine the disturbance range of induced stress field on fracture morphology, the multiple factors analysis and optimization are an important inspect. Setting the induced stress control range (ISCR) as the evaluation objective, it can be defined as the combined response of the peak value of induced stress difference and stress steering distance, taking them as double evaluation indexes. Six uncertain fracture parameters with a reasonable range are used to fit the response of evaluation indexes. Through the finite element numerical simulation method, the induced stress field around the fractures can be calculated and the evaluation indexes are described quantitatively. Applying the multi-factor orthogonal test method, combined the matrix analysis model with the fuzzy comprehensive evaluation model, these fracture parameters are evaluated and optimized. The results show that the influence factors on the ISCR in the descending order are determined as follows: fracture height, fracture net pressure, Poisson's ratio, maximum and minimum horizontal stress ratio, fracture spacing and its number. According to the multi-factors sensitivity analysis results, the selected levels of different factors are optimized and the combination of parameters optimization is screened out. It provides a theoretical basis to control the reservoir stress field for the optimization of the field fracturing treatment.
\end{abstract}

Keywords Hydraulic fracturing $\cdot$ Induced stress control range (ISCR) $\cdot$ Influencing factors $\cdot$ Sensitivity evaluation $\cdot$ Multiparameter optimization

\section{Introduction}

With the increasing consumption of oil and gas resources, the development of unconventional oil and gas resources has entered a white-hot stage. Due to the low porosity and low permeability, the main mean to develop this kind of reservoir is horizontal well fracturing at present, and its essence is to form a complex fracture network achieving the economic benefits. The fracture opens against the minimum principal stress and propagates in the direction of the maximum principal stress, so the stress field distribution is the key to controlling the formation characteristics of fractures (Weng et al. 2011; Li et al.

Bing Guan

sygcgb1990@163.com

1 Key Laboratory of Education Ministry for Enhancing Oil and Gas Recovery, Northeast Petroleum University, Daqing, Heilongjiang, China

2 Operating Crew of the Second Oil Production Plant, Daqing Oilfield Co., Ltd., Daqing, Heilongjiang, China
2014). In the process of fracturing, fractured fractures produce induced stress disturbing the distribution of the original in situ stress field, which is also known as the stress shadow. The stress around the existing fracture changes its initial direction, and it has an impact on the formation of the new fracture. When the induced stress difference is greater than or equal to the original stress difference between the maximum and minimum principal stress, the new fracture will deviate from the original path and occur to reorientation (Zeng and Guo 2016).

The induced stress field determines the complexity of the fracture morphology. In order to better explain and analyse the induced stress field, many profound and elaborate researches had been done. The stress distribution around the fracture was studied by Sneddon and Elliott (1946), and its calculation formula in an infinite elastic body was deduced. Warpinski and Branagan (1989) proposed the concept of altered-stress fracturing which means that the stress mutual interference existing between adjacent fractures could lead to the fracture reorientation. Fisher et al. (2004) revealed the effect of stress shadow based on the results of microseismic monitoring in Barnett 
shale gas reservoir and pointed out that the stress shadow contributed to the formation of complex fracture network according to its influence distance. Olson and Dahi-Taleghani (2009) figured out that due to the effect of stress shadow, the width of the middle one in multiple parallel fractures is minimum under the compressive stress generated by the fractures on its both sides. East et al. (2011) identified the reasonable induced stress could promote the formation of fracture network, and Roussel and Sharma (2011) calculated the degree of stress steering and reorientation through simulating the stress interference of one or more effective fractures with three-dimensional numerical model. The mathematical model of the induced stress field was established, and the disturbance law of the influence factors was clarified by Guo et al. (2014). The influence of the stress interference among fractures on the multistage cluster fracturing of horizontal wells was studied by Wu and Olson (2016). According to the principle of fluid-solid coupling, under different initial stress conditions the distribution of stress field around a single fracture was described by Xia et al. (2016), and through the numerical simulation method, the effect of stress shadow among multiple fractures under different influence factors was analysed by Zeng and Yao (2016). The local stress field around the fracture of the horizontal well was studied by Li et al. (2016a), and the change law of the induced stress field around single fracture and stress interference between multiple fractures were analysed and determined.

Most of these researches focused on the change law of the stress around the fractures. However, the researches on the induced stress control range (ISCR) and the effect degree of these different factors can rarely be seen. In this paper, through clarifying the representation of the induced stress field and calculating the peak value of induced stress difference and the stress steering distance under different influence factors, the effect of each influence factors on the ISCR is evaluated with two different analysis models, and the method to determinate the optimal parameter combination under the action of multiple factors is provided. It is the essential prerequisite to determine the stimulated reservoir volume and provides a theoretical basis to the hydraulic fracturing design.

\section{Induced stress field representation}

Analysing the induced stress field of fracturing fracture, its representation model should be established first. In order to simplify the complexity of the model, some assumptions are made as follows:

(1) The formation is homogeneous and isotropic;

(2) The rock is the porous medium in the linear elastic state without its internal micro-cracks;

(3) The changes in mechanical properties caused by the physical and chemical interaction between rocks and fracturing fluids cannot be taken into account;
(4) The additional induced stress caused by the poroelastic properties of the rock matrix and formation temperature is ignored;

(5) The morphology of the fracture is assumed as the PKN model without the influence of the nature fracture.

Based on the above assumptions, the stress analysis around the fracture in the reservoir can be reduced to a twodimensional plane problem. The schematic diagram of $n$ fracture-induced stress at a given point is shown in Fig. 1.

In Fig. 1, the $x$ axis is the direction of the minimum horizontal principal stress $\sigma_{h}$; the $y$ axis is the direction of the maximum horizontal principal stress $\sigma_{H}$; the $z$ axis is the direction of the vertical stress $\sigma_{v}$.

According to the stress superposition principle, the stress induced by the $n$ fractures at the point of $(x, z)$ in $x z$ plane is obtained as Eq. (1) (Yin et al. 2012).

$$
\left\{\begin{array}{c}
\sigma_{z}=\sum_{i=1}^{n}\left\{\begin{array}{r}
-p_{i} \frac{r_{i}}{h_{i}}\left[\frac{\left(h_{i}\right)^{2}}{r_{i 1} r_{i 2}}\right]^{3 / 2} \sin \theta_{i} \sin \left[\frac{3}{2}\left(\theta_{i 1}+\theta_{i 2}\right)\right] \\
-p_{i}\left[\frac{r_{i}}{\left(r_{i 1} r_{i 2}\right)^{1 / 2}} \cos \left(\theta_{i}-\frac{1}{2} \theta_{i 1}-\frac{1}{2} \theta_{i 2}\right)-1\right]
\end{array}\right\} \\
\sigma_{x}=\sum_{i=1}^{n}\left\{\begin{array}{c}
p_{i} \frac{r_{i}}{h_{i}}\left[\frac{\left(h_{i}\right)^{2}}{r_{i 1} r_{i 2}}\right]^{\frac{3}{2}} \sin \theta_{i} \cdot \sin \left[\frac{3}{2}\left(\theta_{i 1}+\theta_{i 2}\right)\right] \\
-p_{i}\left[\frac{r_{i}}{\left(r_{i 1} r_{i 2}\right)^{1 / 2}} \cos \left(\theta_{i}-\frac{1}{2} \theta_{i 1}-\frac{1}{2} \theta_{i 2}\right)-1\right]
\end{array}\right\} \\
\sigma_{y}=v\left(\sigma_{z}+\sigma_{x}\right) \\
=-2 v \sum_{i=1}^{n}\left\{p _ { i } \left[\frac{r_{i}}{\tau_{z x}}=\sum_{i=1}^{n}\left\{-p_{i} \frac{r_{i}}{h_{i}}\left[\frac{\left(h_{i}\right)^{2}}{r_{i 1} r_{i 2}}\right]^{\frac{3}{2}} \sin \theta_{i} \cos \left[\frac{3}{2}\left(\theta_{i 1}+\theta_{i 2}\right)\right]\right\}\right.\right.
\end{array}\right\}
$$

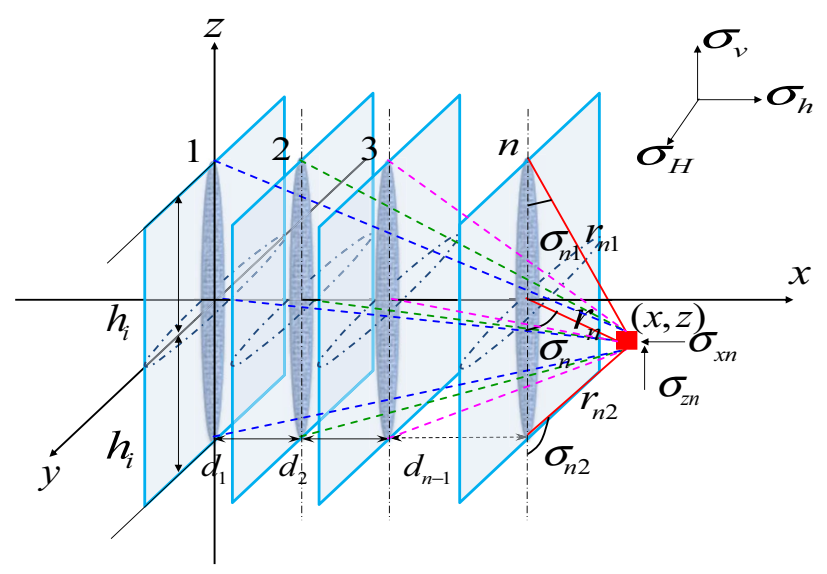

Fig. 1 Fracture-induced stress schematic diagram 
where $\sigma_{z}, \sigma_{x}$ and $\sigma_{y}$ are the normal stress components of the induced stress generated by $n$ fracture at the point of $(x, z)$, respectively, $\mathrm{MPa} ; \tau_{z x}$ is its shear stress component, MPa; $p_{i}$ is the fluid pressure inside the $i$ th fracture, MPa; $r_{i}, r_{i 1}$ and $r_{i 2}$ are the distance of the middle, top and bottom ends of $i$ th fracture to the point of $(x, z)$, respectively, $\mathrm{m} ; \theta_{i}, \theta_{i 1}$ and $\theta_{i 2}$ are the angles between them, ${ }^{\circ} ; \boldsymbol{v}$ is the rock Poisson's ratio; $h_{i}$ is the half height of $i$ th fracture, $\mathrm{m}$.

Based on Fig. 1, the relations of each geometric parameter are as follows:

$$
\begin{aligned}
& \left\{\begin{array}{l}
r_{i}=\sqrt{\left(x-\sum_{i=1}^{n} d_{i-1}\right)^{2}+z^{2}} \\
r_{i 1}=\sqrt{\left(x-\sum_{i=1}^{n} d_{i-1}\right)^{2}+\left(h_{i}-z\right)^{2}} \\
r_{i 2}=\sqrt{\left(x-\sum_{i=1}^{n} d_{i-1}\right)^{2}+\left(h_{i}+z\right)^{2}}
\end{array}\right. \\
& \left\{\begin{array}{l}
\theta_{i}=\arctan \left[-\left(x-\sum_{i=1}^{n} d_{i-1}\right) / z\right] \\
\theta_{i 1}=\arctan \left[\left(x-\sum_{i=1}^{n} d_{i-1}\right) /\left(h_{i}-z\right)\right] \\
\theta_{i 2}=\arctan \left[-\left(x-\sum_{i=1}^{n} d_{i-1}\right) /\left(h_{i}+z\right)\right]
\end{array}\right.
\end{aligned}
$$

where $d_{i-1}$ is the distance of $i(i=1,2, \ldots, n)$ fracture to the $z$ axis, and $d_{0}=0, \mathrm{~m}$.

So the induced stress difference between the direction of the minimum and the maximum horizontal principal stress can be obtained as follows:

$\Delta \sigma^{\prime}=\sigma_{h}^{\prime}-\sigma_{H}^{\prime}=\sigma_{x}-\sigma_{y}$

Combined the induced stress difference of the existing fracture with the initial stress field, the morphological feature of the new fracture can be determined (Liu et al. 2016).

\section{Induced stress control range (ISCR) definition}

A force has three elements, including point of action, magnitude and direction. Similarly, for the induced stress field, there are also two aspects of the magnitude and direction needed to analyse. The research purpose of the induced stress field is to determine the effect of the induced stress field generated by the fractured fracture on the subsequent fracturing. It can be summarized as the degree of influence and the range of influence, which, respectively, refer that how much difference between the induced stress field and the original stress field and that how far the original stress field is disturbed. We define it as the induced stress control range (ISCR), which is a spatial definition, as shown in Fig. 2. Its evaluation indexes are specifically quantified as the peak value of induced stress difference and the stress steering distance.

\section{Evaluation indexes calculation}

The research on fracture with complex morphology is not easy to carry out, so an assumption is done as mentioned that the fracturing fracture is open and is a single main channel perpendicular to the minimum principal stress direction without any branch. Selecting the plane where the maximum horizontal principal stress and the minimum horizontal principal stress are located, the induced stress field of this horizontal plane is analysed in two dimensions. In order to more intuitively and effectively analyse the influence law of multiple factors on the induced stress field by fracturing and considering the fluid-solid coupling, the finite element numerical simulation is adopted. And combined with the calculation mathematical model of induced stress, its numerical model has been validated in previous publications (Roussel and Sharma 2011; Li et al. 2016b), which can be employed to obtain the distribution of induced stress field accurately. The main governing equations are as follows:

\section{(1) Fluid flow}

To describe fluid flow, the Darcy velocity is inserted into an equation of continuity

$\nabla\left[-\frac{\kappa}{\mu} \nabla p\right]=0$

where $\kappa$ is the permeability, $\mathrm{m}^{2} ; \mu$ is the dynamic viscosity, $\mathrm{Pa} \cdot \mathrm{s} ; p$ is the pore pressure of the reservoir, MPa.

For the flow boundaries, the change of the fluid pressure is from the fracture to the reservoir edge. Because the fracture is the only exit for the fluid, there is no flow to or from connecting fracture segments. It should be noted that the

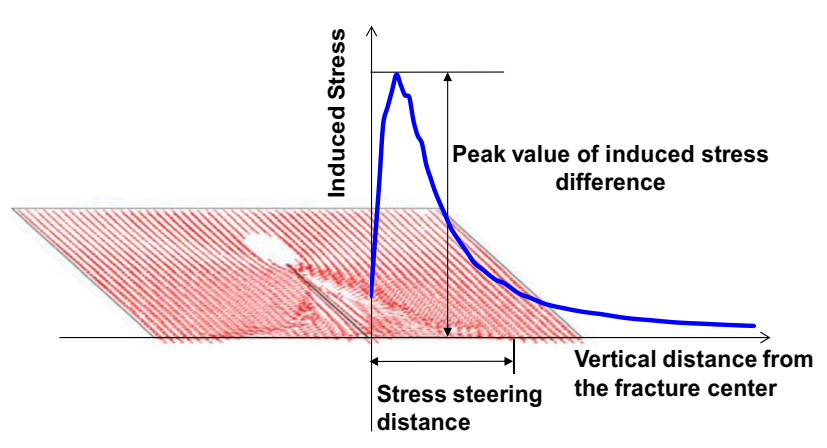

Fig. 2 The ISCR spatial definition diagram 
induced stress field caused by wellbore is not considered. In summary:

$p=p_{0} \quad \partial \Omega$ reservoir

$\mathbf{n} \cdot\left(-\frac{\kappa}{\mu} \nabla p\right)=0 \quad \partial \Omega$ connecting segments

$p=p_{i} \quad \partial \Omega$ fracture

where $\mathbf{n}$ is the normal vector to the boundary.

\section{(2) Solid deformation}

The deformation of reservoir rocks is regarded as a static process, and its stress equilibrium equation is

$\nabla \cdot \sigma+\mathbf{F}=0$

where $\sigma$ denotes the stress tensor, MPa, and $\mathbf{F}$ is the component of body force, $\mathrm{MPa} / \mathrm{m}$. The stress tensor is augmented by the pressure load due to changes in pore pressure. In this model, the Biot-Willis coefficient is equal to one.

The components of the strain tensor $\varepsilon$ depend on the displacement vector $\mathbf{u}$, which has directional components $u$ and $v$. Its strain-displacement equation is

$$
\begin{aligned}
\varepsilon_{x} & =\frac{\partial u}{\partial x} \\
\varepsilon_{y} & =\frac{\partial v}{\partial y} \\
\varepsilon_{x y} & =\frac{1}{2}\left(\frac{\partial u}{\partial y}+\frac{\partial v}{\partial x}\right)
\end{aligned}
$$

where $\varepsilon$ is the strain tensor, and $\mathbf{u}$ is the displacement vector, $\mathrm{m}$. The tensors $\sigma$ and $\varepsilon$ are linearly related by Hooke's law.

For the boundary conditions, the fracture stress filed simulation constrains movement at all external boundaries. The fracture opening is free to deform. In summary:

$u=v=0 \quad \partial \Omega \quad$ reservoir

$v=0 \quad \partial \Omega$ connecting segments

\section{free $\partial \Omega$ fracture}

Based on the above equation and boundary conditions, the finite element numerical simulation can be carried out. To analyse the induced stress around a fracture, a geological model with a magnitude of $2000 \times 1000 \mathrm{~m}$ and a fracture with half height of $100 \mathrm{~m}$ is established. Using the relative parameters shown in Table 1 to set the constraints and boundary loads for the model, the stress distribution of the fracturing fracture in the reservoir is simulated. Intercepting the disturbed stress area around the fracture near horizontal well, the results can be achieved shown in Figs. 3 and 4.
Table 1 Relative parameters in simulation

\begin{tabular}{ll}
\hline Parameters & Value \\
\hline Horizontal maximum principal stress $\sigma_{H} / \mathrm{MPa}$ & 40 \\
Horizontal minimum principal stress $\sigma_{h} / \mathrm{MPa}$ & 32 \\
Original formation pressure $p_{0} / \mathrm{MPa}$ & 10 \\
Porosity $\phi / \%$ & 0.04 \\
Permeability $\kappa / \mathrm{m}^{2}$ & $5.398 \times 10^{-18}$ \\
Poisson's ratio $\nu$ & 0.25 \\
Young's modulus $E / \mathrm{MPa}$ & 23,000 \\
Fracture net pressure $p_{i} / \mathrm{MPa}$ & 10 \\
Fracture half-height $h_{i} / \mathrm{MPa}$ & 100 \\
\hline
\end{tabular}

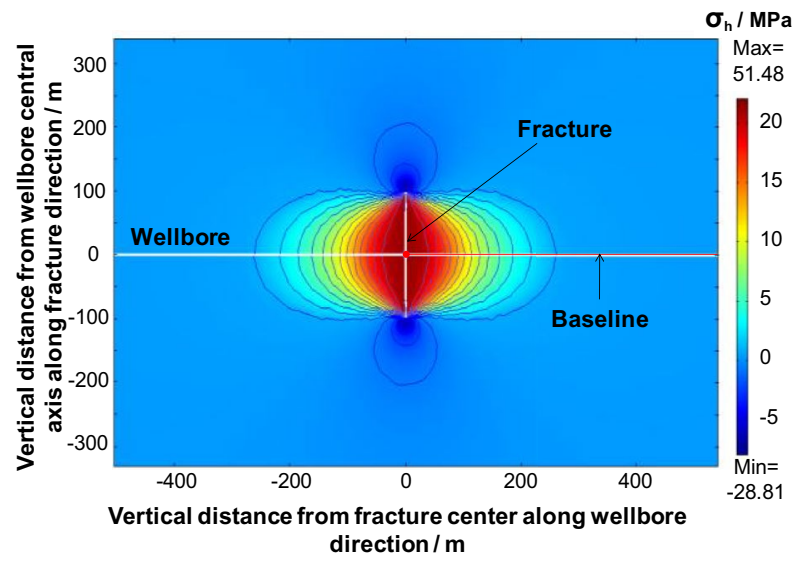

Fig. 3 The minimum horizontal principal stress distribution of a single fracture in induced stress field on horizontal plane (the numbers in the colour bar indicate the change of stress value)

In order to reveal the change law of induced stress around the fracture more clearly, selecting the centre of the fracture as the origin point, the straight line through origin point and perpendicular to the fracture wall is regarded as the research baseline (shown as the red solid line in Fig. 3). Then the induced stress on the baseline can be analysed. Considering the fracture height and net pressure as variables in different fracturing schemes influencing the induced stress, in order to avoid the confusion of variable values and to clarify the magnitude and location of induced stress extremum more clearly, the vertical distance from fracture centre and the induced stress is treated dimensionlessly based on the fundamental parameters of fracture including fracture height $h_{i}$ and net pressure $p_{i}$. Then the change rule curve of induced stress in one-dimensional linear is drawn as shown in Fig. 5. In which the horizontal coordinate represents the distance change of induced stress in the form of a fracture half-height multiple, and the vertical coordinate represents the magnitude change of induced stress in the form of a fracture net pressure multiple. 


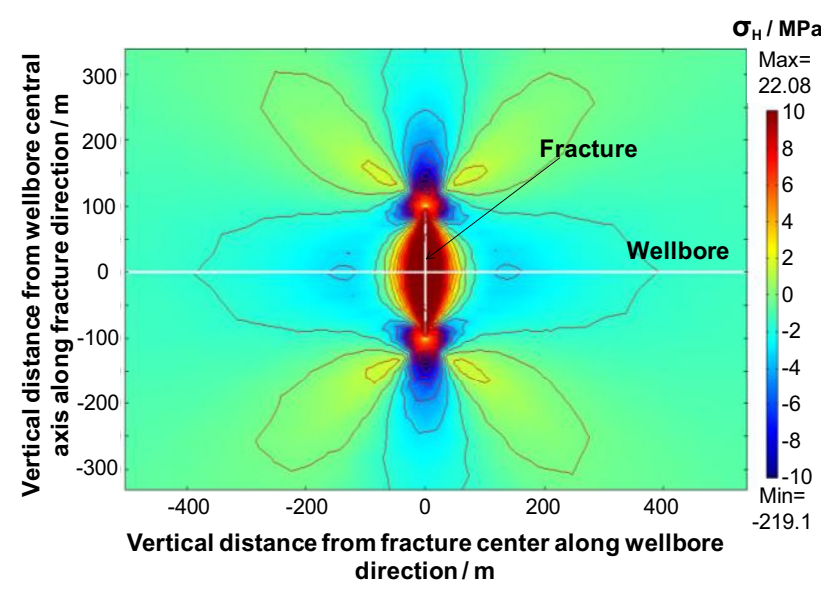

Fig. 4 The maximum horizontal principal stress distribution of a single fracture in induced stress field on horizontal plane (the numbers in the colour bar indicate the change of stress value)

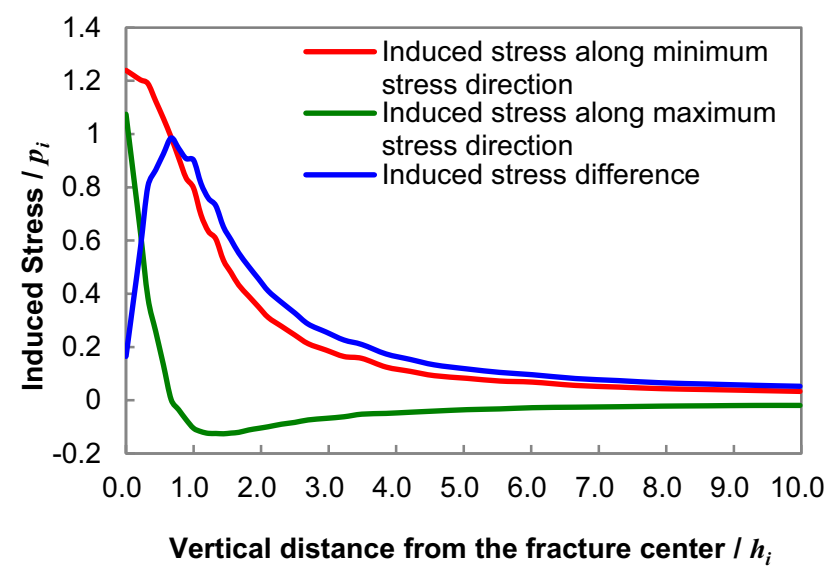

Fig. 5 Induced stress distribution around a fracture along the direction of wellbore central axis

The induced stress not only changes the magnitude of the original stress distribution, but its direction in a certain distance has also changed. In order to define the range of stress steering, the concept of stress steering distance is proposed, which indicates the maximum distance from the artificial fracture surface to the edge of stress steering area along the wellbore direction (Yue and Guo 2012). The stress steering area represents the change of the original stress direction, which deviates from its original path until the principal stress reverses. When the induced stress difference $\Delta \sigma^{\prime}$ produced by the fracture is greater than or equal to the original stress difference $\left(\sigma_{H}-\sigma_{h}\right)$, the principal stress reversal will occur. Then with the increase in the distance perpendicular to the artificial fracture surface, the effect of induced stress field disappears gradually, and the direction of principal stress restores to its original state. So the stress steering distance can be defined as the distance from the artificial fracture surface to the point of the principal stress reversal along the wellbore direction. Taking the distribution direction of maximum principal stress in formation after fracture disturbance as an example, the schematic diagram of the stress steering distance in induced stress field is shown in Fig. 6. It can be observed that under the action of induced stress field of artificial fracture, a significant phenomenon of maximum horizontal principal stress steering occurred near the fracture. The maximum horizontal principal stress around the fracture changes from the original direction along the fracture to the direction perpendicular to the fracture, while that far from the fracture remains in the original direction. The criterion to measure the stress steering distance is to determine the point of the principal stress reversal. Its distance is about a half height of the fracture and it can be calculated by the finite element numerical simulation.

Describing a change of the induced stress should include two aspects: magnitude and direction. So the peak value of induced stress difference and the stress steering distance is recognized as the evaluation indexes to determine the ISCR. And the larger the two indexes are, the greater the ISCR is.

\section{Influence factors determination}

According to the above analysis, the factors influencing the distribution of the induced stress field can be determined, such as the maximum and minimum horizontal stress ratio, Poisson's ratio, fracture net pressure, fracture height, fracture spacing, fracture number and so on. Under different influencing factors, the ISCR is different. In order to analyse the relationship between these factors and the ISCR, subsequent research is carried out.

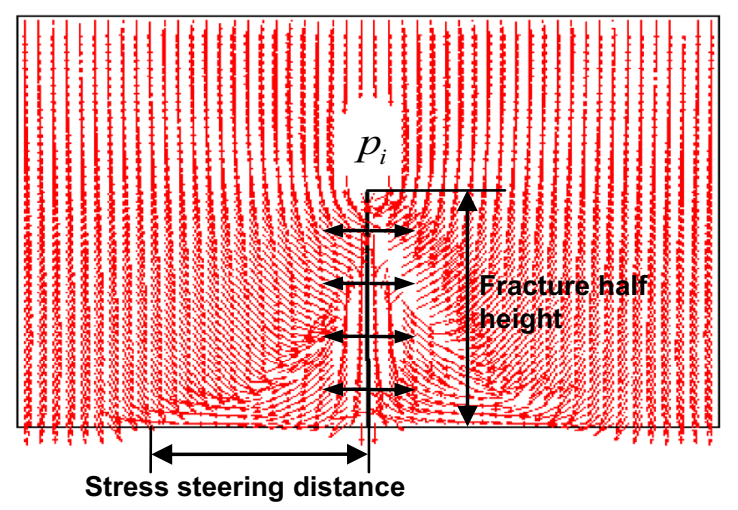

Fig. 6 The schematic diagram for defining the stress steering distance by the change of the direction of the maximum horizontal principal stress in induced stress field

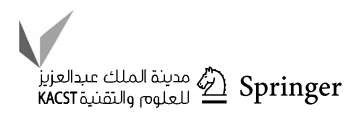




\section{Orthogonal experimental design}

The sensitivity of the influence factor represents the influence degree on the ISCR. In order to analyse the combined action of multiple factors effectively, the orthogonal test design (Fang et al. 2014; Yao et al. 2012) method is applied. There are six influence factors, including the maximum and minimum horizontal stress ratio, Poisson's ratio, fracture net pressure, fracture height, fracture spacing and its number. And five levels of each factor are selected, as shown in the example Table 2. According to the number of test factors and the level of factors, the orthogonal test can be designed as the orthogonal table of $L_{25}\left(5^{6}\right)$, as shown in Table 3. Through the finite element numerical simulation method, the stress steering distance $D_{\mathrm{s}}$ and the peak value of induced stress difference $\Delta \sigma_{\max }$ in horizontal direction can be calculated, as shown in Figs. 7 and 8.

\section{Multi-factors sensitivity results analysis}

By the range results analysis of the orthogonal experiment, the multiple objective functions are obtained, including the stress steering distance $D_{\mathrm{s}}$ and the peak value of induced stress difference $\Delta \sigma_{\max }$. In order to determine the influence of multi-factors on the ISCR, two different methods are used to deal with the evaluation results of multiple indexes.

\section{Orthogonal test matrix analysis model}

According to the orthogonal test data structure, a three-layer structure model can be established, including the layer of evaluation indexes, factors and levels (Wang 2018).

In each layer of evaluation indexes, there are $l$ factors and each factor includes $m$ levels. The mean value of the test index in the $j$ th level of one factor $A_{i}$ is $k_{i j}$. If the larger the result of evaluation indexes is better, $K_{i j}=k_{i j}$. And if the smaller the result of evaluation indexes is better, $K_{i j}=1 / k_{i j}$.
Then the matrix of evaluation indexes can be obtained as follows:

$$
I=\left[\begin{array}{ccccc}
K_{11} & 0 & 0 & \ldots & 0 \\
K_{12} & 0 & 0 & \ldots & 0 \\
\ldots & \ldots & \ldots & \ldots & \ldots \\
K_{1 m} & 0 & 0 & \ldots & 0 \\
0 & K_{21} & 0 & \ldots & 0 \\
0 & K_{22} & 0 & \ldots & 0 \\
\ldots & \ldots & \ldots & \ldots & \ldots \\
0 & K_{2 m} & 0 & \ldots & 0 \\
\ldots & \ldots & \ldots & \ldots & \ldots \\
0 & 0 & 0 & \ldots & K_{l 1} \\
0 & 0 & 0 & \ldots & K_{l 2} \\
\ldots & \ldots & \ldots & \ldots & \ldots \\
0 & 0 & 0 & \ldots & K_{l m}
\end{array}\right]
$$

In the layer of factors, its matrix of $F_{i}=1 / \sum_{j=1}^{m} K_{i j}$ can be obtained as follows:

$F=\left[\begin{array}{cccc}F_{1} & 0 & \ldots & 0 \\ 0 & F_{2} & \ldots & 0 \\ \ldots & \ldots & \ldots & \ldots \\ 0 & 0 & \ldots & F_{l}\end{array}\right]$

In the layer of levels, the range of factor $A_{i}$ is $r_{i}$. There is $L_{i}=r_{i} / \sum_{i=1}^{l} r_{i}$, so the matrix of levels can be obtained as follows:

$L^{T}=\left[\begin{array}{llll}L_{1} & L_{2} & \ldots & L_{l}\end{array}\right]$

The weight matrix of each level in each factor influencing on the evaluation index can be obtained as $\omega=I F L$.

$\omega^{T}=\left[\begin{array}{llll}\omega_{1} & \omega_{2} & \ldots & \omega_{m}\end{array}\right]$
Table 2 Evaluation of each factor under different levels

\begin{tabular}{lllcrrr}
\hline Factors level & $\sigma_{H} / \sigma_{h}$ & $v$ & $p_{i} / \mathrm{MPa}$ & $h_{i} / \mathrm{m}$ & $d_{i-1} / \mathrm{m}$ & $n$ \\
\hline 1 & 1.2 & 0.1 & 5 & 50 & 50 & 2 \\
2 & 1.4 & 0.2 & 10 & 100 & 100 & 3 \\
3 & 1.5 & 0.3 & 15 & 150 & 150 & 4 \\
4 & 1.6 & 0.4 & 20 & 200 & 200 & 5 \\
5 & 1.8 & 0.45 & 25 & 250 & 250 & 6 \\
\hline
\end{tabular}


Table 3 Orthogonal design table

\begin{tabular}{|c|c|c|c|c|c|c|c|c|}
\hline Factor schemes & $\sigma_{H} / \sigma_{h}$ & $v$ & $p_{i} / \mathrm{MPa}$ & $h_{i} / \mathrm{m}$ & $d_{i-1} / \mathrm{m}$ & $n$ & $D_{\mathrm{s}} / \mathrm{m}$ & $\Delta \sigma_{\max } / \mathrm{MPa}$ \\
\hline No. 1 & 1.2 & 0.1 & 5 & 50 & 50 & 2 & 63.0 & 13.42 \\
\hline No. 2 & 1.5 & 0.3 & 5 & 250 & 100 & 6 & 299.3 & 19.22 \\
\hline No. 3 & 1.5 & 0.2 & 10 & 100 & 50 & 5 & 120 & 19.90 \\
\hline No. 4 & 1.8 & 0.45 & 5 & 200 & 150 & 5 & 249.5 & 21.00 \\
\hline No. 5 & 1.8 & 0.3 & 15 & 150 & 50 & 3 & 167.5 & 22.20 \\
\hline No. 6 & 1.4 & 0.1 & 10 & 250 & 150 & 3 & 277 & 16.60 \\
\hline No. 7 & 1.8 & 0.1 & 25 & 100 & 200 & 6 & 119.4 & 20.50 \\
\hline No. 8 & 1.4 & 0.2 & 5 & 150 & 200 & 4 & 182.6 & 16.60 \\
\hline No. 9 & 1.8 & 0.2 & 20 & 250 & 250 & 2 & 252.3 & 21.85 \\
\hline No. 10 & 1.8 & 0.4 & 10 & 50 & 100 & 4 & 56.2 & 22.30 \\
\hline No. 11 & 1.6 & 0.2 & 15 & 50 & 150 & 6 & 56.2 & 19.85 \\
\hline No. 12 & 1.6 & 0.1 & 20 & 150 & 100 & 5 & 163.6 & 24.30 \\
\hline No. 13 & 1.2 & 0.4 & 15 & 250 & 200 & 5 & 306.9 & 19.90 \\
\hline No. 14 & 1.6 & 0.4 & 5 & 100 & 250 & 3 & 136.3 & 20.60 \\
\hline No. 15 & 1.4 & 0.3 & 25 & 50 & 250 & 5 & 55.7 & 21.50 \\
\hline No. 16 & 1.6 & 0.3 & 10 & 200 & 200 & 2 & 240.7 & 20.03 \\
\hline No. 17 & 1.2 & 0.45 & 10 & 150 & 250 & 6 & 184.6 & 21.01 \\
\hline No. 18 & 1.5 & 0.4 & 25 & 150 & 150 & 2 & 170.9 & 22.81 \\
\hline No. 19 & 1.2 & 0.2 & 25 & 200 & 100 & 3 & 219.3 & 21.60 \\
\hline No. 20 & 1.4 & 0.4 & 20 & 200 & 50 & 6 & 254.8 & 22.88 \\
\hline No. 21 & 1.2 & 0.3 & 20 & 100 & 150 & 4 & 104.4 & 21.00 \\
\hline No. 22 & 1.6 & 0.45 & 25 & 250 & 50 & 4 & 306.2 & 21.36 \\
\hline No. 23 & 1.4 & 0.45 & 15 & 100 & 100 & 2 & 117.3 & 20.38 \\
\hline No. 24 & 1.5 & 0.1 & 15 & 200 & 250 & 4 & 216.4 & 18.40 \\
\hline No. 25 & 1.5 & 0.45 & 20 & 50 & 200 & 3 & 56.6 & 20.67 \\
\hline Mean 1 & 175.6 & 167.9 & 186.1 & 57.5 & 182.3 & 168.8 & The stre & \\
\hline Mean 2 & 177.5 & 166.1 & 175.7 & 119.5 & 171.1 & 171.3 & & \\
\hline Mean 3 & 172.6 & 173.5 & 172.9 & 173.8 & 171.6 & 173.2 & & \\
\hline Mean 4 & 180.6 & 185.0 & 166.3 & 236.1 & 181.2 & 179.1 & & \\
\hline Mean 5 & 169.0 & 182.8 & 174.3 & 288.3 & 169.1 & 182.9 & & \\
\hline Range & 11.62 & 18.93 & 19.80 & 230.8 & 13.24 & 14.03 & & \\
\hline Mean 1 & 19.39 & 18.64 & 18.17 & 19.55 & 19.95 & 19.70 & \multirow{6}{*}{\multicolumn{2}{|c|}{$\begin{array}{l}\text { The peak value of induced stress difference } \\
\Delta \sigma_{\max }\end{array}$}} \\
\hline Mean 2 & 19.59 & 19.96 & 19.97 & 20.48 & 21.56 & 20.33 & & \\
\hline Mean 3 & 20.20 & 20.79 & 20.15 & 21.38 & 20.25 & 19.93 & & \\
\hline Mean 4 & 21.23 & 21.70 & 22.14 & 20.78 & 19.54 & 21.32 & & \\
\hline Mean 5 & 21.57 & 20.88 & 21.55 & 19.79 & 20.67 & 20.69 & & \\
\hline Range & 2.18 & 3.05 & 3.97 & 1.84 & 2.02 & 1.62 & & \\
\hline
\end{tabular}

In which $\omega_{1}=K_{11} F_{1} L_{1}$, it can be explained from two aspects. The first part is $K_{11} F_{1}=K_{11} / \sum_{j=1}^{m} K_{i j}$, which represents the ratio between the index value of 1 st level and the index values sum of all levels in $A_{1}$ factor. The second part is $L_{1}=r_{1} / \sum_{i=1}^{l} r_{i}$, which represents the ratio between the range value of $A_{1}$ factor and the range values sum. The numerical product of the two parts can not only reflect the influence degree of 1 st level in $A_{1}$ factor on the index value, but also can reflect the magnitude of the range in $A_{1}$ factor. Other factors and levels can be obtained in this way.

Through calculating, combined the two evaluation indexes of the stress steering distance $D_{\mathrm{s}}$ and the induced stress difference $\Delta \sigma_{\max }$, the weight effects of each level in each factor on the comprehensive evaluation indexes are determined as shown in Table 4 and its 3D display is shown in Fig. 9.

According to the weight results, the primary and secondary order of each factor influencing on the evaluation 


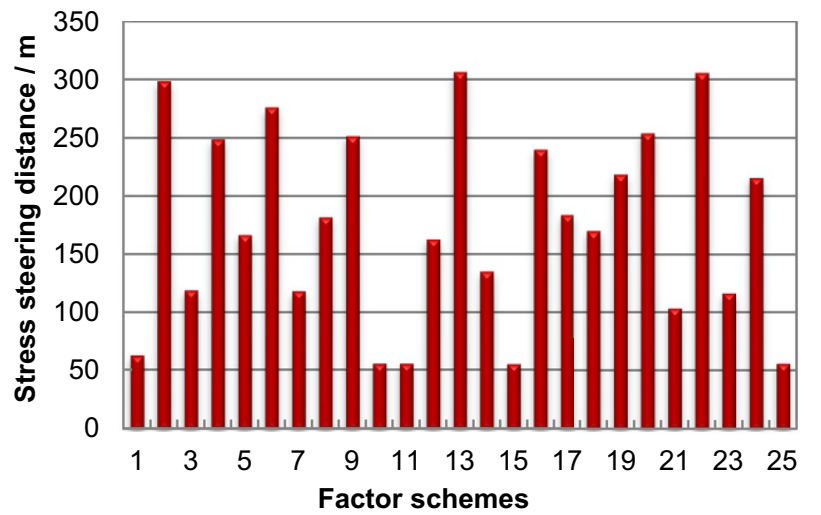

Fig. 7 The results of the stress steering distance under different factor schemes

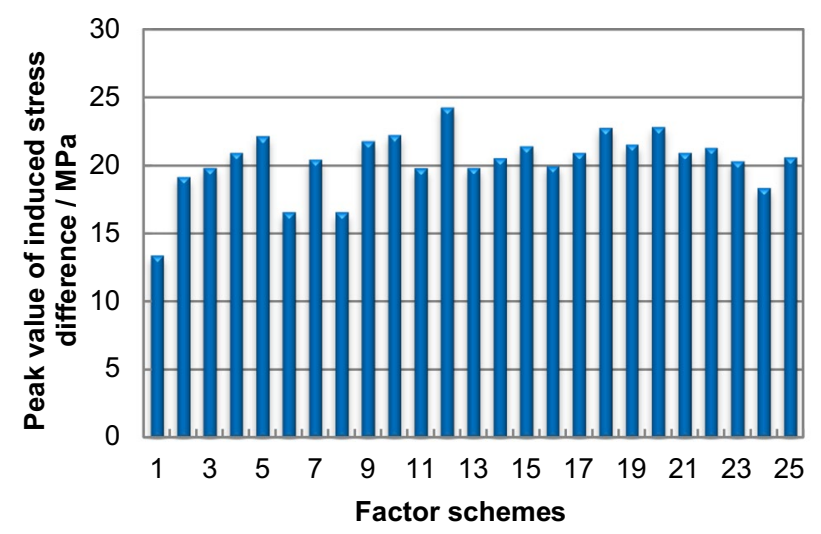

Fig. 8 The results of the peak value of induced stress difference under different factor schemes

indexes is $h_{i}>p_{i}>v>\sigma_{H} / \sigma_{h}>d_{i-1}>n$, and the optimization combination of multi-factors is $\sigma_{H} / \sigma_{h}=1.6 ; v=0.4$; $p_{i}=20 ; h_{i}=250 ; d_{i-1}=100 ; n=5$.

\section{Fuzzy comprehensive evaluation model}

Fuzzy comprehensive evaluation is the method that makes an overall evaluation of the objects restricted by many factors, in which the qualitative evaluation is transformed into quantitative evaluation by utilizing the fuzzy mathematics (Fang et al. 2013; Moayyedian et al. 2018). In this paper, the fuzzy comprehensive evaluation is used to confirm the ISCR caused by various influence factors. By taking the stress steering distance $D_{\mathrm{s}}$ and the peak value of induced stress difference $\Delta \sigma_{\max }$ shown in Table 3 as the evaluation indexes, and the 25 groups of test results are used as the evaluation objects, the main steps and calculation results of fuzzy comprehensive evaluation are introduced as follows.

\section{(1) Membership function establishment}

The membership function is established which mappings the value of $D_{\mathrm{s}}$ and $\Delta \sigma_{\max }$ to membership grade. Since the value of $D_{\mathrm{s}}$ and $\Delta \sigma_{\max }$ is proportional to the ISCR, the membership function is presented as follows:

$r_{i j}=\frac{Y_{i j}-\max \left(Y_{i}\right)}{\max \left(Y_{i}\right)-\min \left(Y_{i}\right)}, \quad i=1,2 ; j=1,2, \ldots 25$

In the function, $Y_{1}$ represents the $D_{\mathrm{s}}$ and $Y_{2}$ represents the $\Delta \sigma_{\text {max }}$. Therefore, the fuzzy relation matrix is shown as follows:

$R=\left(r_{i j}\right)_{m \times n}$

(2) Weight allocation set establishment

The weight allocation set reflects the importance of each index, and the weight allocation set $A$ is shown as follows:

$A=\left[\begin{array}{ll}\alpha_{1} & \alpha_{2}\end{array}\right]$

where $\alpha_{1}+\alpha_{2}=1$.

(3) Calculation of fuzzy comprehensive evaluation

Through the fuzzy transformation, the value of fuzzy comprehensive evaluation can be obtained with the function as follows:

$B=A \circ R$

In this paper, "o" means the operator $M(\bullet, \oplus)$, which is defined as follows:
Table 4 Weight calculation results of each level in each factor under comprehensive evaluation indexes

\begin{tabular}{lllllll}
\hline Factor level & $\sigma_{H} / \sigma_{h}$ & $v$ & $p_{i} / \mathrm{MPa}$ & $h_{i} / \mathrm{m}$ & $d_{i-1} / \mathrm{m}$ & $n$ \\
\hline 1 & 0.0179 & 0.0249 & 0.0309 & 0.0366 & 0.0179 & 0.0151 \\
2 & 0.0181 & 0.0262 & 0.0329 & 0.0636 & $\mathbf{0 . 0 1 8 7}$ & 0.0155 \\
3 & 0.0184 & 0.0273 & 0.0331 & 0.0874 & 0.0179 & 0.0153 \\
4 & $\mathbf{0 . 0 1 9 4}$ & $\mathbf{0 . 0 2 8 6}$ & $\mathbf{0 . 0 3 5 5}$ & 0.1137 & 0.0176 & $\mathbf{0 . 0 1 6 2}$ \\
5 & 0.0194 & 0.0277 & 0.0350 & $\mathbf{0 . 1 3 5 4}$ & 0.0181 & 0.0160 \\
Optimum & $\sigma_{H} / \sigma_{h}-4$ & $v-4$ & $p_{i}-4$ & $h_{i}-5$ & $d_{i-1}-2$ & $n-4$ \\
\hline
\end{tabular}

The optimal result values of each factor are marked in bold 


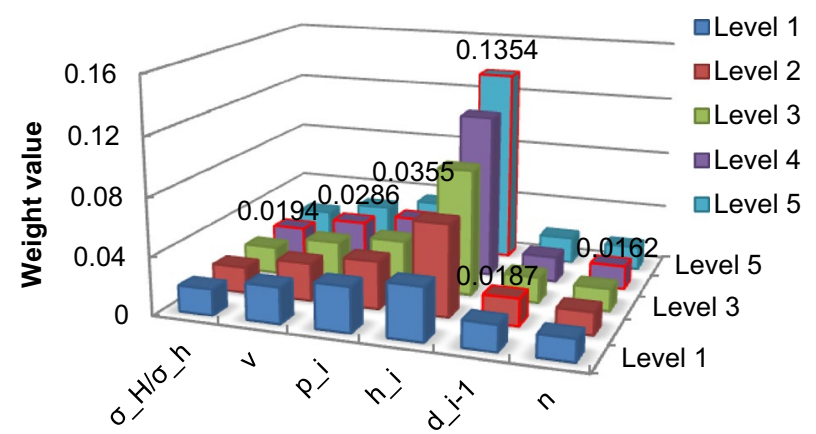

Influence factors

Fig. 9 Weight calculation results of each level in each factor in 3D view

Table 5 Membership degree and comprehensive evaluation value of influence factors

\begin{tabular}{lcllll}
\hline Factor level & $D_{\mathrm{s}} / \mathrm{m}$ & $\Delta \sigma_{\mathrm{max}} / \mathrm{MPa}$ & $R(:, 1)$ & $R(:, 2)$ & $B$ \\
\hline No. 1 & 63.0 & 13.42 & 0.029 & 0 & 0.015 \\
No. 2 & 299.3 & 19.22 & 0.97 & 0.533 & 0.751 \\
No. 3 & 120 & 19.90 & 0.256 & 0.596 & 0.426 \\
No. 4 & 249.5 & 21.00 & 0.771 & 0.697 & 0.734 \\
No. 5 & 167.5 & 22.20 & 0.445 & 0.807 & 0.626 \\
No. 6 & 277 & 16.60 & 0.881 & 0.292 & 0.587 \\
No. 7 & 119.4 & 20.50 & 0.254 & 0.651 & 0.452 \\
No. 8 & 182.6 & 16.60 & 0.505 & 0.292 & 0.399 \\
No. 9 & 252.3 & 21.85 & 0.783 & 0.775 & 0.779 \\
No. 10 & 56.2 & 22.30 & 0.002 & 0.816 & 0.409 \\
No. 11 & 56.2 & 19.85 & 0.002 & 0.591 & 0.296 \\
No. 12 & 163.6 & 24.30 & 0.43 & 1 & 0.715 \\
No. 13 & 306.9 & 19.90 & 1 & 0.596 & 0.798 \\
No. 14 & 136.3 & 20.60 & 0.321 & 0.66 & 0.49 \\
No. 15 & 55.7 & 21.50 & 0 & 0.743 & 0.371 \\
No. 16 & 240.7 & 20.03 & 0.736 & 0.608 & 0.672 \\
No. 17 & 184.6 & 21.01 & 0.513 & 0.698 & 0.605 \\
No. 18 & 170.9 & 22.81 & 0.459 & 0.863 & 0.661 \\
No. 19 & 219.3 & 21.60 & 0.651 & 0.752 & 0.702 \\
No. 20 & 254.8 & 22.88 & 0.793 & 0.869 & 0.831 \\
No. 21 & 104.4 & 21.00 & 0.194 & 0.697 & 0.445 \\
No. 22 & 306.2 & 21.36 & 0.997 & 0.73 & 0.863 \\
No. 23 & 117.3 & 20.38 & 0.245 & 0.64 & 0.442 \\
No. 24 & 216.4 & 18.40 & 0.64 & 0.458 & 0.549 \\
No. 25 & 56.6 & 20.67 & 0.004 & 0.666 & 0.335 \\
\hline & & & & &
\end{tabular}

$b_{j}=\sum_{i=1}^{m} a_{i} r_{i j}$
(4) Main effect analysis

By doing statistical analysis and normalization, the effect of different factor values on the comprehensive evaluation results can be obtained. The $C_{i}$ is proposed to represent the $i$ th influence factor, where $i=1,2, \ldots, 6 . \tilde{C}_{i}$ is introduced to show the results of main effect analysis.

$\tilde{C}_{i}=\left(\frac{\sum b_{i 1}}{T}, \frac{\sum b_{i 2}}{T}, \frac{\sum b_{i 3}}{T}, \frac{\sum b_{i 4}}{T}, \frac{\sum b_{i 5}}{T}\right) \quad i=1,2, \ldots, 6$

where $T=\sum_{i=1}^{25} b_{i}, \sum b_{i k}, k=1,2, \ldots, 5$ means the sum of comprehensive evaluation values corresponded to the $k$ th level of $i$ th influence factor.

Based on the previous steps, the membership degree of influence factors and the comprehensive evaluation value is obtained as shown in Table 5. Besides, the results of main effect analysis are shown in Table 6 and its 3D display is shown in Fig. 10.

Finally, the order of the influencing factors by the principle of maximum membership degree can be determined as $h_{i}>v>\cdot>\sigma_{H} / \sigma_{h}>d_{i-1}>n$, and the optimization combination of multi-factors is $\sigma_{H} / \sigma_{h}=1.6 ; v=0.4 ; p_{i}=20$; $h_{i}=250 ; d_{i-1}=100$ and $n=5$.

Compared the evaluation results of two models, there are some small differences, but the primary and secondary effect order of factors on the evaluation indexes are determined quantitatively. In which the fracture half-height $h_{i}$ is the most sensitive factors, the Poisson's ratio $v$ and fracture net pressure $p_{i}$ is better sensitive, followed by the maximum and minimum horizontal stress ratio $\sigma_{H} / \sigma_{h}$ and the spacing $d_{i-1}$, and the least sensitive factor is the fracture number $n$. The combination of parameters optimization is $\sigma_{H} / \sigma_{h}=1.6$; $v=0.4 ; p_{i}=20 ; h_{i}=250 ; d_{i-1}=100$ and $n=5$.

\section{Conclusions}

1. A spatial definition of the induced stress field generated by fractured fracture is proposed as the induced stress control range (ISCR), and two evaluation indexes to describe the ISCR are specifically quantified as the peak value of induced stress difference and the stress steering distance. According to the mathematical model of the horizontal induced stress field, six main controlling factors influencing on the ISCR are revealed, including the maximum and minimum horizontal stress ratio, Poisson's ratio, fracture net pressure, fracture height, fracture spacing and fracture number.

2. By the means of multi-factor orthogonal test, the influence degree of six factors on the ISCR is identified, and combined with the matrix analysis model and the fuzzy 
Table 6 Results of main effect analysis

\begin{tabular}{lllllll}
\hline Level & $C_{1}$ & $C_{2}$ & $C_{3}$ & $C_{4}$ & $C_{5}$ & $C_{6}$ \\
\hline 1 & 0.184 & 0.166 & 0.171 & 0.102 & 0.198 & 0.184 \\
2 & 0.188 & 0.186 & 0.193 & 0.162 & $\mathbf{0 . 2 1 6}$ & 0.196 \\
3 & 0.195 & 0.205 & 0.194 & 0.215 & 0.195 & 0.191 \\
4 & $\mathbf{0 . 2 1 8}$ & $\mathbf{0 . 2 2 9}$ & $\mathbf{0 . 2 2 3}$ & 0.250 & 0.190 & $\mathbf{0 . 2 1 5}$ \\
5 & 0.215 & 0.214 & 0.219 & $\mathbf{0 . 2 7 1}$ & 0.200 & 0.210 \\
Optimum & $\sigma_{H} / \sigma_{h}-4$ & $v-4$ & $p_{i}-4$ & $h_{i}-5$ & $d_{i-1}-2$ & $n-4$ \\
\hline
\end{tabular}

The optimal result values of each factor are marked in bold

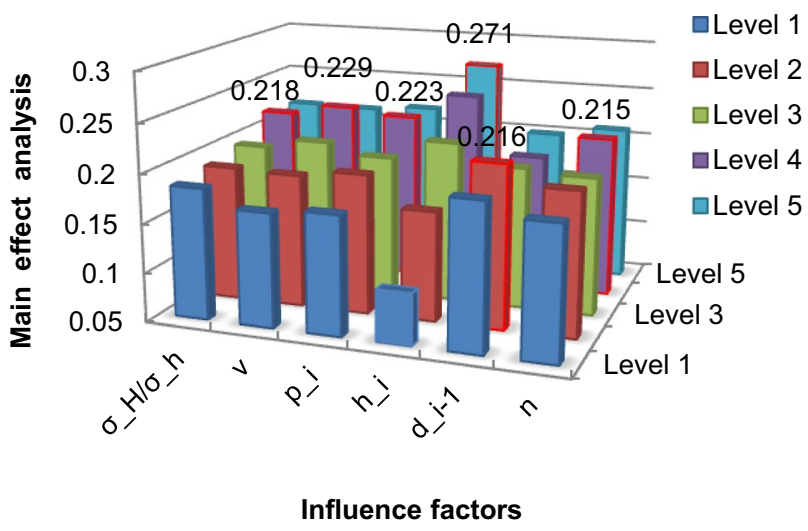

Fig. 10 Main effect analysis results of each level in each factor in 3D view

comprehensive evaluation model, the sensitivity of each factor to the ISCR is evaluated comprehensively. The influence order in the descending is as follows: fracture height, fracture net pressure, Poisson's ratio, the maximum and minimum horizontal stress ratio, fracture spacing and its number.

3. According to the multi-factors sensitivity results analysis, the optimal parameter combination of the fracturing treatment design is preferred. One thing must be emphasized that the results depend on the selection of the levels for different factors in a certain. The research model in this paper is based on some assumptions, and the actual fracturing conditions are more complex, involving more influencing factors. So in the actual situation, the process of parameters optimization can be adjusted for the construction need. It only provides a method to determine the optimum parameters of the fracture-induced stress field under the combined action of multiple factors.

Acknowledgements The authors would like to thank the support provided by the National Natural Science Foundation of China (Grant Nos. 51874098 and 51504067) and Northeast Petroleum University Innovation Foundation for Postgraduate (Grant No. JYCX_CX02_2018).
Open Access This article is distributed under the terms of the Creative Commons Attribution 4.0 International License (http://creativeco mmons.org/licenses/by/4.0/), which permits unrestricted use, distribution, and reproduction in any medium, provided you give appropriate credit to the original author(s) and the source, provide a link to the Creative Commons license, and indicate if changes were made.

\section{References}

East L, Soliman MY, Augustine J (2011) Methods for enhancing far-field complexity in fracturing operations. SPE Prod Oper 26(3):291-303

Fang JJ, Guan RF, Ma JQ et al (2013) Optimization of fabrication parameters to prepare tea catechin-loaded liposomes by fuzzy orthogonality. Key Eng Mater 531-532:458-464

Fang HS, Tian J, Wang S et al (2014) Numerical optimization of czochralski sapphire single crystal growth using orthogonal design method. Cryst Res Technol 49(5):323-330

Fisher MK, Heinze JR, Harris CD et al (2004) Optimizing horizontal completion techniques in the Barnett shale using microseismic fracture mapping. In: SPE annual technical conference and exhibition. https://doi.org/10.2118/90051-MS

Guo JC, Yin J, Zhao ZH (2014) Feasibility of formation of complex fractures under cracks interference in shale reservoir fracturing. Chin J Rock Mech Eng 33(8):1589-1596

Li J, Guo BY, Feng Y (2014) An analytical solution of fracture-induced stress and its application in shale gas exploitation. J Energy Res Technol 163(2):1-6

Li SB, Guan B, Zhang LG et al (2016a) Local stress field disturbance law of horizontal well fracturing. Pet Geol Recov Effic 23(6):112-119

Li W, Li LC, Tang CA (2016b) Numerical simulation research on mechanism of induced stress perturbation between parallel fractures in horizontal wells. Nat Gas Geo Sci 27(11):2043-2053

Liu SY, Lou YS, Wu H et al (2016) Optimization of multi-cluster fracturing model under the action of induced stress in horizontal wells. J Eng Sci Technol Rev 9(2):59-65

Moayyedian M, Abhary K, Marian R (2018) Optimization of injection molding process based on fuzzy quality evaluation and Taguchi experimental design. CIRP J Manuf Sci Technol 21:150-160

Olson JE, Dahi-Taleghani A (2009) Modeling simultaneous growth of multiple hydraulic fractures and their interaction with natural fractures. In: SPE hydraulic fracturing technology conference. https://doi.org/10.2118/119739-MS 
Roussel NP, Sharma MM (2011) Optimizing fracture spacing and sequencing in horizontal-well fracturing. SPE Prod Oper 26(2):173-184

Sneddon LN, Elliott HA (1946) The opening of a Griffith crack under internal pressure. Q Appl Math 4(3):262-267

Wang Y, Huo X (2018) Multiobjective optimization design and performance prediction of centrifugal pump based on orthogonal test. Adv Mater Sci Eng 15:1-10

Warpinski NR, Branagan PT (1989) Altered-stress fracturing. J Pet Technol 41(9):990-997

Weng DW, Lei Q, Xu Y et al (2011) Network fracturing techniques and its application in the field. Acta Pet Sin 32(2):280-284

Wu K, Olson JE (2016) Mechanisms of simultaneous hydraulic-fracture propagation from multiple perforation clusters in horizontal wells. SPE J 21(3):1000-1008

Xia L, Zeng YW, Jin L et al (2016) Research on influence of initial horizontal principal stress on stress shadow. J Rock Mech Eng 35(S1):2819-2825
Yao CJ, Lei GL, Ma JY et al (2012) Laboratory experiment, modeling and field application of indigenous microbial flooding. J Pet Sci Eng 90-91:39-47

Yin J, Guo JC, Zeng FH (2012) Perforation spacing optimization for staged fracturing of horizontal well. Pet Drill Tech 40(5):67-71

Yue YC, Guo JC (2012) Fluid-solid coupling analysis of reorientation mechanism of refracturing. Rock Soil Mech 32(10):3189-3193

Zeng FH, Guo JC (2016) Optimized design and use of induced complex fractures in horizontal wellbores of tight gas reservoirs. Rock Mech Rock Eng 49(4):1411-1423

Zeng QD, Yao J (2016) Numerical simulation of fracture network generation in naturally fractured reservoirs. J Nat Gas Sci Eng 30:430-443

Publisher's Note Springer Nature remains neutral with regard to jurisdictional claims in published maps and institutional affiliations. 